\title{
Efficiency Analysis of Pump-controlled Motor System of Hydraulic Machinery Continuously Variable Transmission for Agricultural Machinery
}

\author{
Mingxi Bao ${ }^{1}$, Xiangdong $\mathrm{Ni}^{1,2, *}$ and Shiwei Kang ${ }^{1}$ \\ ${ }^{1}$ School of Mechanical and Electrical Engineering, Shihezi University, Xin Jiang, China \\ ${ }^{2}$ Key Laboratory of Northwest Agricultural Equipment, Ministry of Agriculture, Shihezi University, Shihezi 832003, China
}

\begin{abstract}
In order to improve the efficiency of Hydraulic Machinery Continuously Variable Transmission (HMCVT), studying the efficiency of its pump-controlled motor system is of great significance to promote the practical application of HMCVT in agricultural machinery. By analyzing the HMCVT transmission principle and the driving parameters of medium and large vehicles, determine the HMCVT transmission scheme that meets the actual requirements. The simulation model of the whole HMCVT system was established by SimulationX software, and the relationship curves between different engine speeds and HMCVT output speeds were given. And determine the effect of the pump-controlled motor system on the response parameters of HMCVT under different volumetric efficiency.
\end{abstract}

\section{Introduction}

A large number of researches have been conducted on HMCVT at home and abroad, but the research objects mainly focus on wind power generation systems and tractors, but there are few related researches on medium and large agricultural machinery. Huang GQ designed a HMCVT with variable speed input and constant speed output for wind power generation system, and simulated its dynamics [1]. Yoo, Young Rak designed an HMCVT power transmission system with a combination of helical gears and planetary gears for an 8-ton medium-duty forklift, calculated the specifications of complex helical and planetary gear drives, analyzed the bending and compressive stress of the gears, and optimized them [2]. According to the basic principle of power distribution, Zhang ZM established the speed, torque, power and efficiency equations of HMCVT to meet the requirements of tractor use [3]. Aiming at the driving demand of agricultural machinery, this research group developed equal-difference HMCVT, and explored the influence of volumetric efficiency of hydraulic speed regulation system of pump-controlled motor on HMCVT, so as to better study the overall performance of HMCVT.

\section{The HMCVT's Struction Principle}

Hydro-mechanical continuously variable transmission is an integrated device that combines hydraulic stepless speed regulation with efficient transmission of mechanical gears. The output power of the engine is divided into two ways by the gear pair, and one is the pump-motor volume speed regulation circuit. All the way is the planetary compound gear train, and then the power is output backward through the confluence device and transmitted to the transmission mechanism [4,5]. The structure principle is shown in Fig. 1.

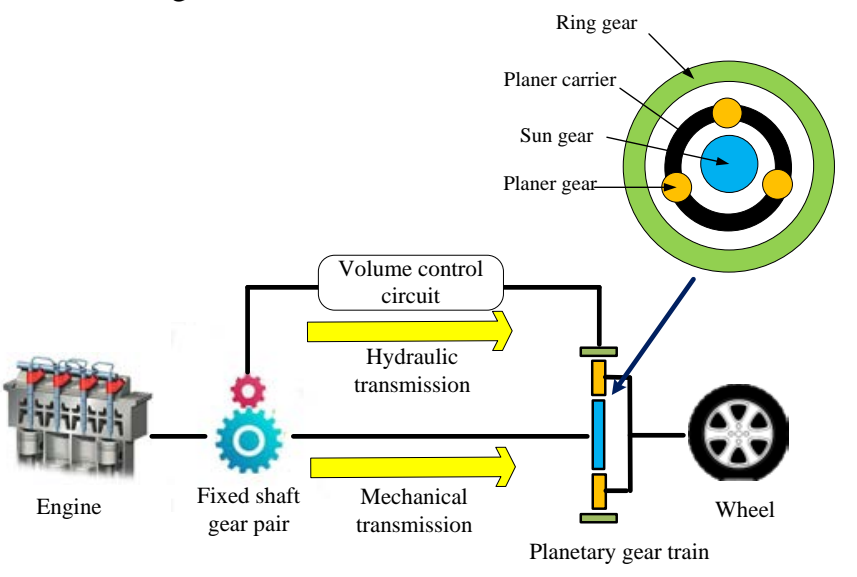

Fig.1. Hydraulic power split structure schematic

\footnotetext{
다responding author: nxd_mac@shzu.edu.cn
} 


\section{The HMCVT's Transmission Scheme}

This research is based on medium and high-power vehicles based on their driving needs and working conditions, independently establishing a transmission scheme, determining HMCVT parameters, and trial-producing prototypes. The basic parameters of HMCVT-adaptive cotton pickers are proposed: HMCVT operating speed range is $0-25 \mathrm{~km} / \mathrm{h}$, with an engine power requirement of $100 \mathrm{~kW}$ and a rated speed of $2200 \mathrm{rpm}$.

This study uses a differential-mechanical continuously variable transmission with constant torque and constant speed, which is mainly composed of a two-way variable pump-controlled constant-motor hydraulic speed control system, a compound planetary gear train, and clutch and brake shift mechanisms. Among them, the hydraulic mechanical continuously variable transmission uses a fixed-shaft gear pair to split the input torque, and the planetary differential compound gear train combines the rotational speeds of the two-degree-of-freedom mechanism. The power input is supplied by the engine. The HMCVT's transmission scheme was shown in Fig. 2. The transmission forward gear is divided into pure hydraulic $\mathrm{H}$ gear, hydraulic mechanical HM1 gear and hydraulic mechanical HM2 gear; reverse gear includes R gear.

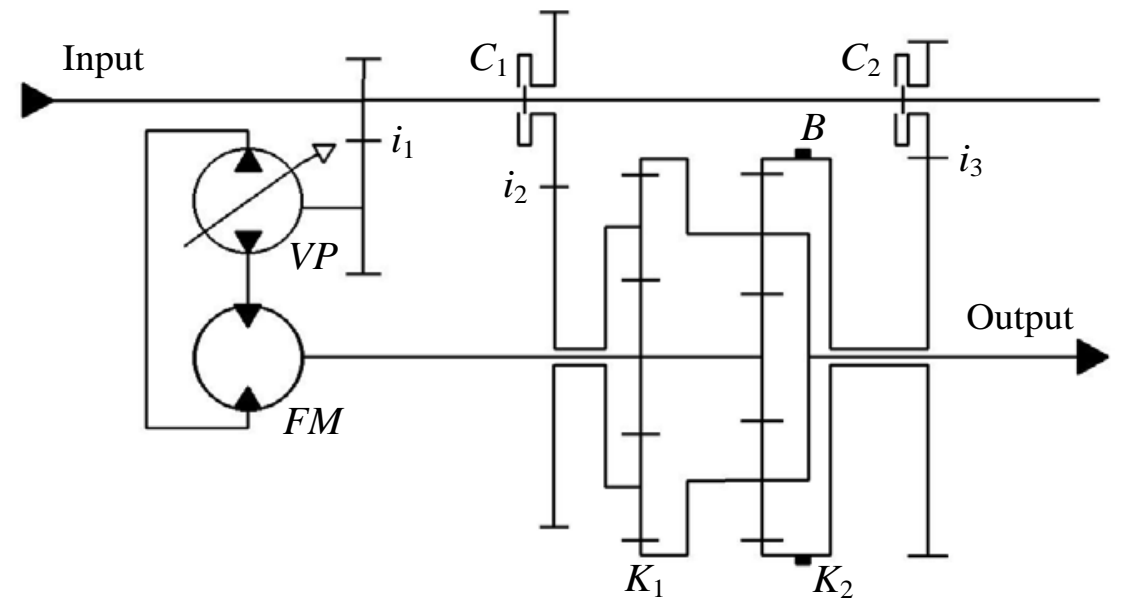

Fig.2. Transmission scheme of hydro mechanical CVT

In this study, a John Deere-JD4945HCP29 diesel engine, HPV-02 high-pressure two-way variable displacement pump, HMF-02 quantitative motor, brake, wet clutch, CZ magnetic powder brake, ZJ speed rotation sensor and TR-1 torque speed measuring instrument were used. And WLK-5A program-controlled power supply. The parameters of gear ratio of HMCVT transmission scheme are: $\mathrm{i} 1=0.5854 ; \mathrm{i} 2=1.3452, \mathrm{i} 3=0.3776$; $\mathrm{k} 1=3.56$; $\mathrm{k} 2=$ 2.56. $\mathrm{K} 1$ is the characteristic parameter of the front planetary gear pair, K2 is the characteristic parameter of the rear planetary gear pair, and its value is the ratio of the number of ring gear teeth to the number of sun gear teeth.

\section{Simulation Model}

Engine, two-way variable displacement pump-controlled fixed-motor hydraulic speed control system, planetary gear train mechanism, clutch / brake hydraulic system) were simulated. According to the established HMCVT complete system simulation model, the HMCVT speed characteristics, the response characteristics of the two-way variable-pump constant-volume motor system, the oil-charging characteristics of the clutch / brake hydraulic system, and the shift smoothness can be studied. The simulation model of HMCVT was shown in Fig. 3.

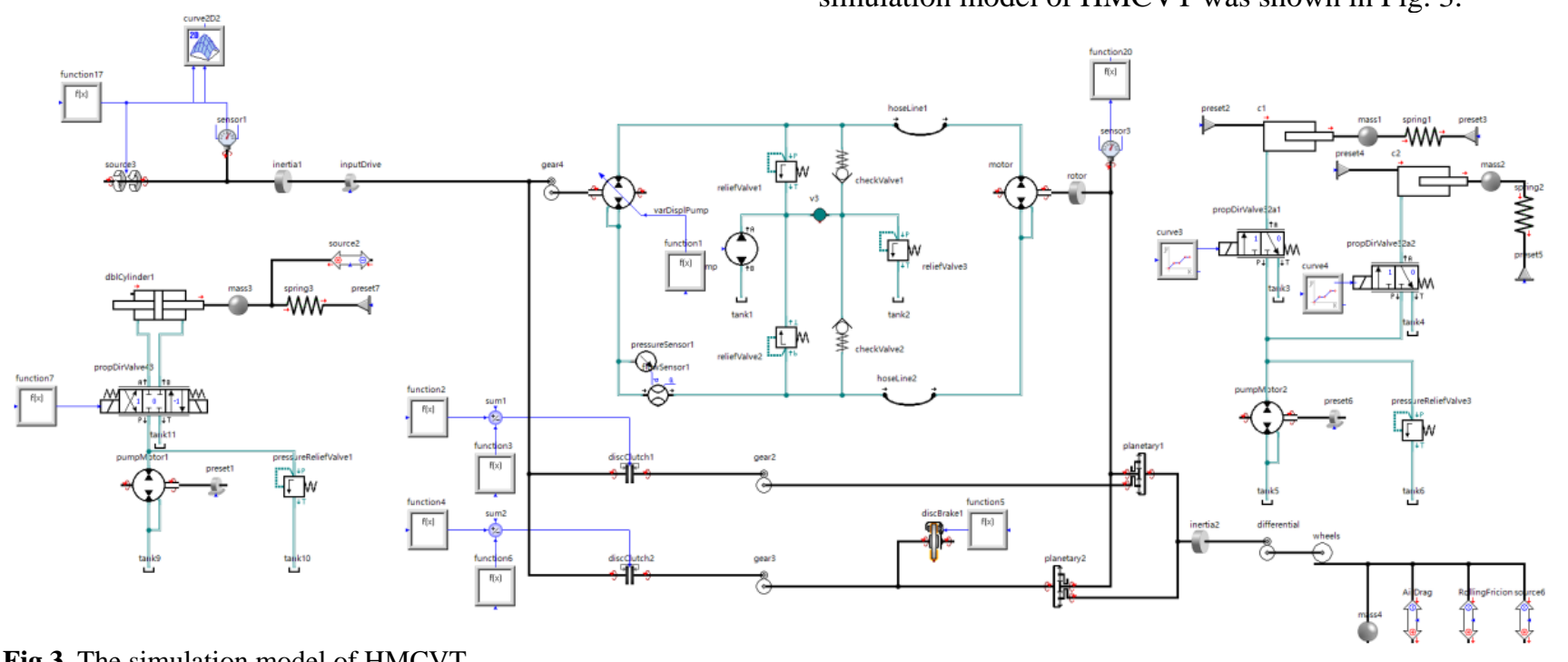

Fig.3. The simulation model of HMCVT 
Control clutch C1, clutch C2 disconnect, and combine the brakes to make the HMCVT work in pure hydraulic $\mathrm{H}$ gear. The displacement of the variable pump is controlled by adjusting the range of the forward and reverse deviation buttons (forward deviation) and displacement ratio in the measurement and control system $(0 \sim 1)$. When the displacement ratio is adjusted to the maximum, that is, when the displacement ratio $=1$, at this time, in conjunction with the clutch $\mathrm{C} 1$, the clutch $\mathrm{C} 2$ continues to be disconnected, the brake is disconnected, and the HMCVT is switched from the pure hydraulic $\mathrm{H}$ gear to the hydraulic mechanical HM1 gear. In the HM1 gear of the hydraulic machine, the displacement ratio varies from 1 to -1 . When the displacement ratio is adjusted to the minimum, that is, when the displacement ratio $=-1$, the HMCVT is switched from the hydraulic mechanical HM1 gear to the hydraulic mechanical HM2 gear. In the HM2 gear of the hydraulic machine, the displacement ratio varies from -1 to 1 . When the engine speed is $1200 \mathrm{rpm}, 1600 \mathrm{rpm}, 2200 \mathrm{rpm}$, the relationship curve of HMCVT output speed was shown in Fig. 4.

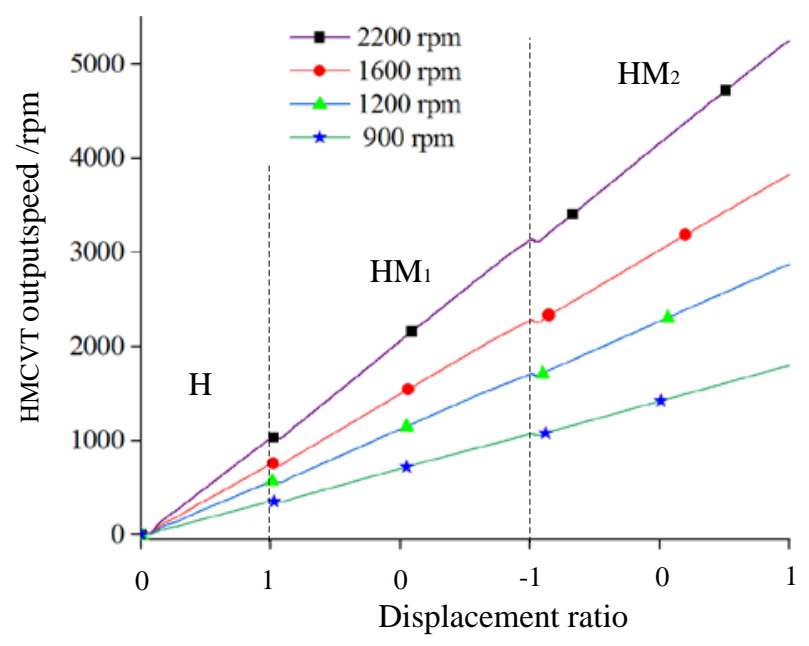

Fig.4. HMCVT output speed

\section{Efficiency Analysis}

The oil leakage and compressibility of the two-way variable displacement pump-controlled fixed-speed motor hydraulic speed control system have a certain effect on the output speed of the fixed-speed motor during gear shifting. Therefore, taking the shift from the HM1 gear of the hydraulic machine to the HM2 gear of the hydraulic

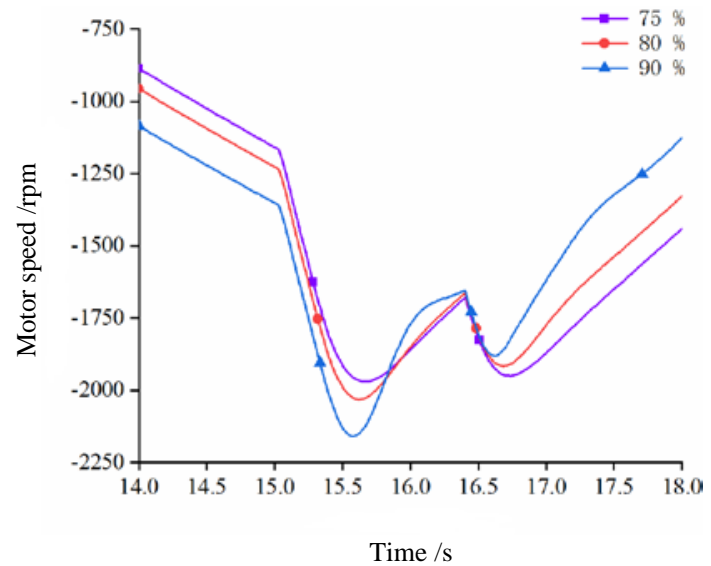

Fig.5. Motor output speed machine as an example, a further simulation analysis is performed on the volumetric efficiency of the two-way variable pump controlled quantitative motor system. The clutch control signal is a control curve of oil pressure and return spring. Here we take the three cases of volume efficiency of the pump-motor $90 \%, 80 \%, 75 \%$ respectively, and the mechanical efficiency is fixed at $90 \%$.

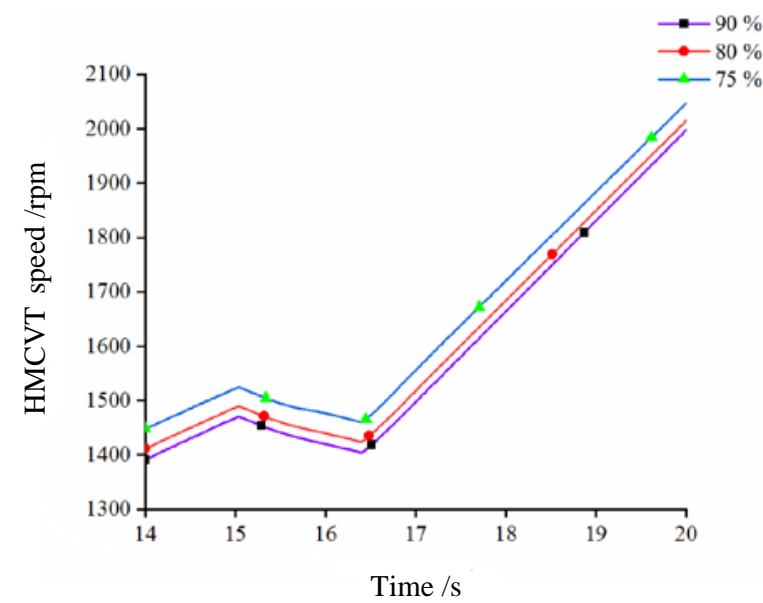

Fig.6. HMCVT output speed 


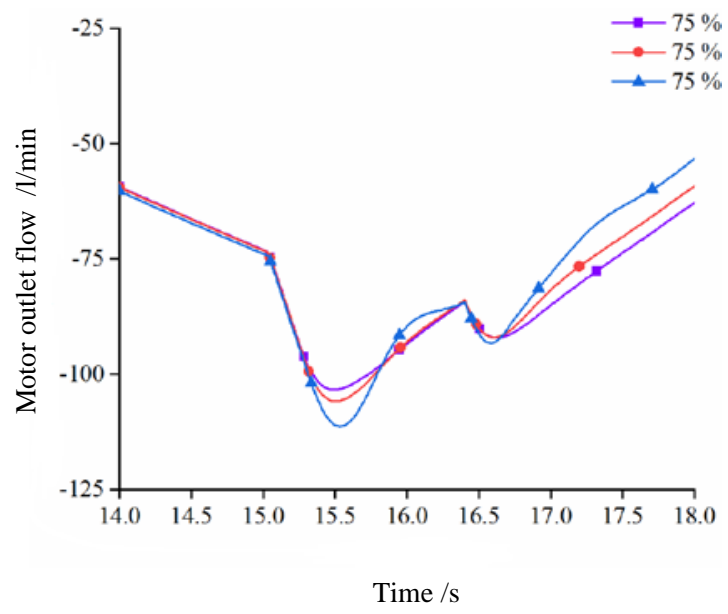

Fig.7. Motor flow

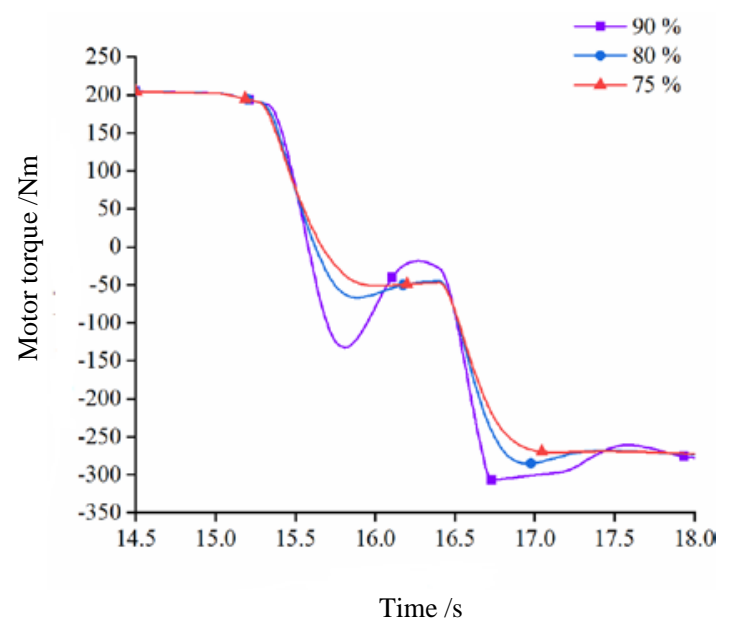

Fig.9. Motor output torque

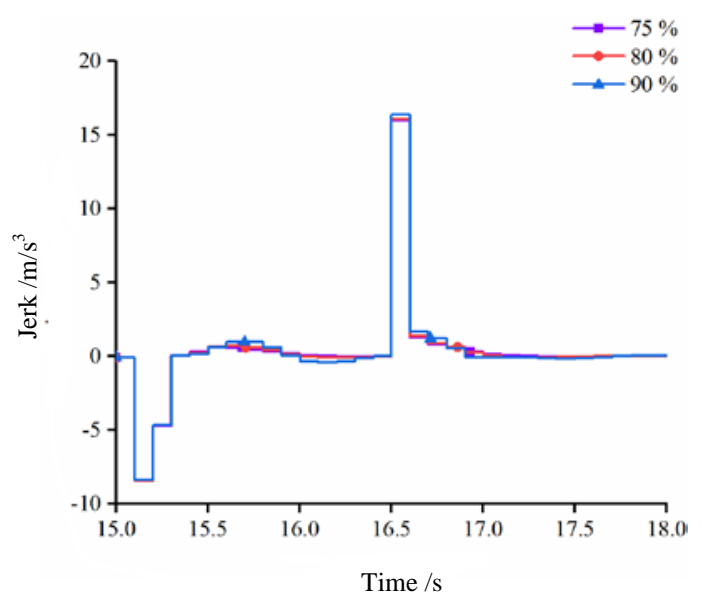

Fig.11. HMCVT jerk

From Fig. 5, Fig. 7, and Fig. 8, the output speed and outlet flow of the fixed-volume motor and the speed difference between the clutch master and slave disks are different before and after the shift. Before the shift, with the increase of volumetric efficiency, the larger the decrease in output speed, motor outlet flow and speed difference, but after the shift is completed, the larger the volumetric efficiency, the increase in speed and speed of motor outlet flow and speed difference will increase. The greater the

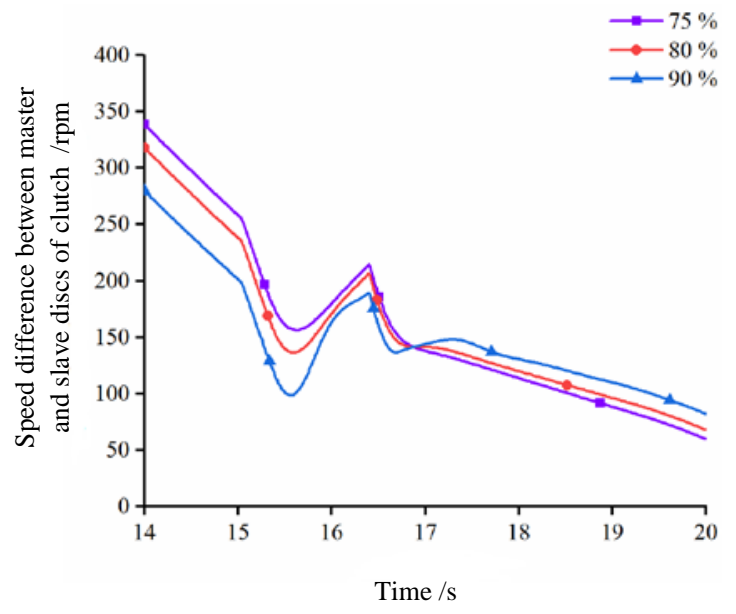

Fig.8. Clutch master and slave disc speed difference

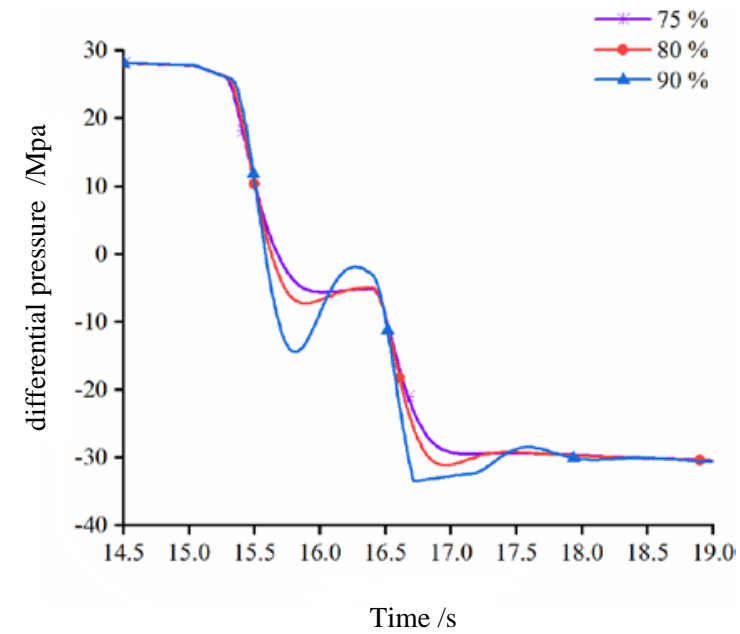

Fig.10. Motor differential pressure

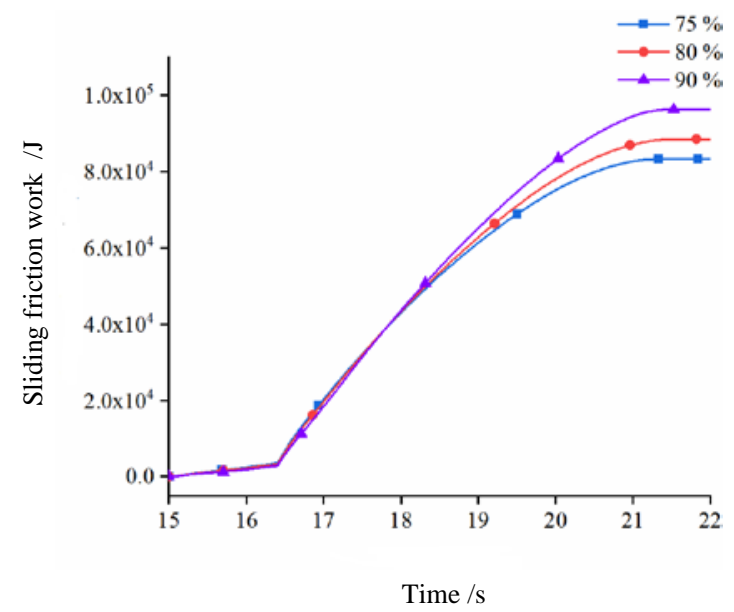

Fig.12. Sliding friction work

speed drop of the fixed motor before and after the shift, the greater the fluctuation in the speed of the HMCVT output shaft. From Fig. 6, the trend of HMCVT output speed in the process of shifting is the same. As can be seen from Fig. 9 and 10, the greater the volumetric efficiency, the greater the decrease in torque and pressure difference during the shifting process, and the faster the lifting speed. It can be known from Fig.11 that the volumetric efficiency has a certain impact on the impact, but the impact is not 
significant. It can be known from Fig.12 that the friction work of the clutch decreases as the volumetric efficiency decreases.

\section{Summary}

The simulation model of HMCVT was established to analyze the relationship between HMCVT output speed and displacement ratio. Taking the hydraulic mechanical HM1 to HM2 as an example, the volume efficiency of the two-way variable pump-controlled quantitative motor system is further simulated and analyzed. When the volume efficiency is $90 \%, 80 \%, 75 \%$, analyze the changes of parameters, such as the output speed and outlet flow rate of the quantitative motor, the velocity difference, the HMCVT output speed, the torque, the pressure difference, the jerk and the sliding friction work

\section{References}

1. Huang, G., Characteristics Investigation for Hydro-Mechanical Compound Transmission in Wind Power System, in Communications in Computer and Information Science, K. Li, et al., K. Li, et al.^Editors.
2018. p. 24-35.

2. Yoo, Y.R., A Strength Analysis of Gear Train for Hydro-Mechanical Continuously Variable Transmission. The International Journal of Advanced Culture Technology, 2018. 6(3): p. 163-172.

3. Zhang, Z. Analysis of Main Characteristics of Hydro-mechanical Continuously Variable Transmission, in AER-Advances in Engineering Research, P.S. Wei, P.S. Wei^Editors. 2016. p. 88-92.

4. Zhang, M., Z. Zhou and L. Xu, TRANSMISSION EFFICIENCY RESEARCH OF MULTI-RANGE HYDRO-MECHANICAL CVT. 2016.

5. Jiancheng Wan, Yaao Zhou, Min Wang, Ming Jiang, and Yong Ma, "Finite Element Simulations and Experimental Research of Grip Clamps of Expanded Diameter Half-Hard Aluminum Conductors Composite Core Reinforced," International Journal of Materials, Mechanics and Manufacturing vol. 7, no. 6, pp. 270-275, 2019 